\title{
Research on the Technology of UAV Landing Using Visual Guidance
}

\author{
Jia Li \\ School of Automation Science and Electrical \\ Engineering \\ Beijing University of Aeronautics and Astronautics \\ Beijing, China \\ E-mail:1i825369659@sina.com
}

\author{
Yangzhu Wang \\ School of Automation Science and Electrical \\ Engineering \\ Beijing University of Aeronautics and Astronautics \\ Beijing, China \\ E-mail:wyz_buaa@sohu.com
}

\author{
Yelin Zhang \\ School of Automation Science and Electrical \\ Engineering \\ Beijing University of Aeronautics and Astronautics \\ Beijing, China \\ E-mail:yixingzhe1993@163.com
}

\begin{abstract}
According to the landmark feature, the paper guides UAV precise landing with machine vision technology. Firstly, the author analyzes the principle of visual navigation based on the landmarks. According to the imaging principle of machine vision, the paper uses projection matrix method to solute the conversion relationship between the plane coordinates and the corresponding image coordinates of the landmarks and then estimates the relative position and attitude parameters of UAV. And then, it designs landmarks of different feature types according to the characteristics of projection matrix to improve the precision of navigation. Finally, it deals with the position of the navigation data by Kalman filter and further improves the navigation precision. The simulation which is done by Matlab simulation environment shoot to the simulated landmarks and parameter solution. Experimental results show that different types of landmarks have certain effect on navigation precision, and the navigation precision has been greatly improved by the addition of Kalman filter.
\end{abstract}

Keywords-UAV;visual navigation; projection matrix; kalman filter; matlab

\section{INTRODUCTION}

At present, the application of UAV is becoming more and more extensive. In the military, the UAV has become part of the battlefield, bears the task of investigation and fight; in civil, UAV is used in reconnaissance, disaster relief and scientific research. As the important stage of UAV flight mission, the landing stage has a high requirement for the navigation and control accuracy, so it has been one of the focus of the UAV research field. The autonomous landing of UAV means the UAV is only relying on the airborne navigation equipment and the flight control system to navigate the navigation system and finally realize the UAV safe landing. It is an urgent problem for UAV to realize autonomous flight, especially the autonomous landing of UAV, which is the urgent problem for the development of UAV technology.

The first problem is to obtain accurate navigation information and achieve accurate guidance for UAV. At present, the navigation mode is based on the combination of GPS and inertial navigation [1]-[3]. Because GPS signal is easy to interfere, or even shut down, it is urgent to develop other navigation methods to obtain navigation information. With the development of computer vision technology and optoelectronic devices, machine vision is increasingly applied to aircraft navigation.

Visual navigation guides UAV landing mainly by installing in the absence of computer imaging equipment to obtain images of the landing area, through computer vision algorithms [4] to estimate UAV flight state with respect to the location of the landing area and the aircraft automatic landing control, and to reach the purpose of precision landing [5] [6].

In this paper, the landmark based visual navigation technology is studied. The technology combined with preset landmarks, with the help of airborne opto electronic platform and image acquisition and processing module, and the on-board computer, opposite phase of land UAV attitude parameters of solution [7]. According to the characteristics of projection matrix, the different types of landmarks are designed, and the results of the Kalman filter are introduced, and the results are more accurate.

\section{COORDINATE SYSTEM IN VISUAL NAVIGATION}

\section{A. Pixel coordinate system and image coordinate system}

The camera stores the image in digital information, and the pixel value corresponds to the image information. As shown in Fig .1, the coordinates of each pixel $(\mathrm{u}, \mathrm{v})$ are represented by the number of rows and columns of the array.

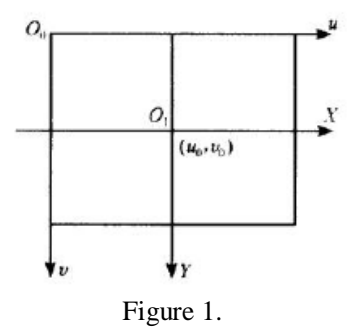


$(\mathrm{u}, \mathrm{v})$ is actually a position in the array, so the camera physical coordinate system is needed. Take the image center $\mathrm{O} 1$ as the origin of the image coordinate system, between the known pixels in the horizontal and vertical to the direction of the physical size of the $d X, d Y$, image pixel coordinates and physical image coordinate system a conversion relation as follows.

$$
\left[\begin{array}{l}
u \\
v \\
1
\end{array}\right]=\left[\begin{array}{ccc}
\frac{1}{d X} & 0 & u_{0} \\
0 & \frac{1}{d Y} & v_{0} \\
0 & 0 & 1
\end{array}\right]\left[\begin{array}{c}
X \\
Y \\
1
\end{array}\right]
$$

\section{B. Camera coordinate system}

In the imaging model of the camera, $\mathrm{O}$ stands for the camera photo center, $x$ shaft and $y$ shaft respectively, and the image of the $\mathrm{X}$ axis and the $\mathrm{Y}$ axis is parallel, $\mathrm{Z}$ axis, and like perpendicular to the plane. The intersection of the optical axis and the image plane is the origin of the image coordinate system, and the Cartesian coordinate system composed of the point $\mathrm{O}$ and $\mathrm{x}, \mathrm{y}, \mathrm{z}$ axis is called the camera coordinate system.

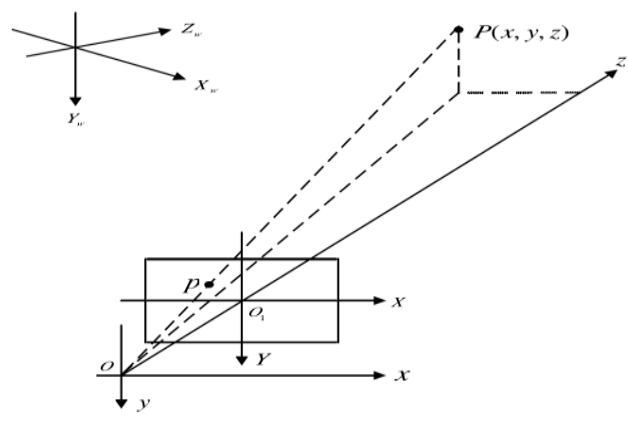

Figure 2.

\section{Conversion relationship of coordinate system}

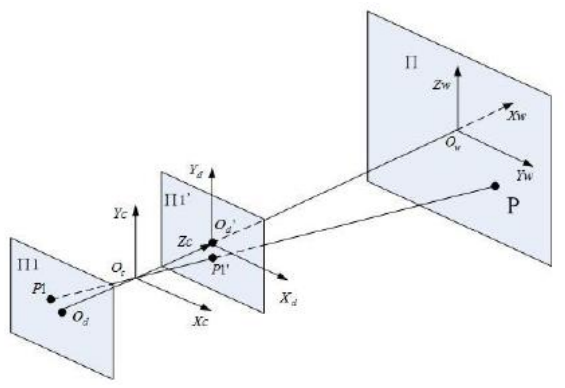

Figure 3.

As shown in Fig.3, $\triangle$ P1OdOc $\backsim \triangle$ POwOc., According to geometrical relationship:

$$
\left\{\begin{array}{l}
\frac{x_{c}}{z_{c}}=\frac{x_{d}}{z_{d}}=\frac{x_{d}}{f} \\
\frac{y_{c}}{z_{c}}=\frac{y_{d}}{z_{d}}=\frac{y_{d}}{f}
\end{array}\right.
$$

As shown in Fig .3, the following transformation relations exist between world coordinate system and camera frame:

$$
\left[\begin{array}{c}
x_{c} \\
y_{c} \\
z_{c} \\
1
\end{array}\right]=\left[\begin{array}{llll}
r_{1} & r_{2} & r_{3} & t_{x} \\
r_{4} & r_{5} & r_{6} & t_{y} \\
r_{7} & r_{8} & r_{9} & t_{z} \\
0 & 0 & 0 & 1
\end{array}\right]\left[\begin{array}{l}
x_{w} \\
y_{w} \\
z_{w} \\
1
\end{array}\right]=\left[\begin{array}{ll}
R & T \\
0 & 1
\end{array}\right]\left[\begin{array}{l}
x_{w} \\
y_{w} \\
z_{w} \\
1
\end{array}\right]
$$

$\mathrm{R}$ as the rotation matrix:

$$
R=\left[\begin{array}{ccc}
\cos \theta \cos \alpha & \sin \theta \cos \alpha & -\sin \alpha \\
\cos \theta \sin \varphi \sin \alpha-\sin \theta \cos \varphi & \cos \theta \cos \varphi+\sin \theta \sin \alpha \sin \varphi & \cos \alpha \sin \varphi \\
\sin \theta \sin \varphi+\cos \theta \sin \alpha \cos \varphi & \sin \theta \sin \alpha \cos \varphi-\cos \theta \sin \varphi & \cos \alpha \cos \varphi
\end{array}\right]
$$

$\mathrm{T}$ as translation matrix:

$$
T=\left[\begin{array}{lll}
T_{X} & T_{Y} & T_{Z}
\end{array}\right]^{T}
$$

\section{Navigation parameter estimation for UAV}

Since RAC calibration method is an important work in computer vision camera calibration, the method firstly radial consistency constraints to solve $\mathrm{Tz}$ camera parameters, then calibrates other parameters[8]. The advantage of this method is that the equation is the linear equation, which reduces the complexity of the parameters, and can be applied to the focal length change.

\section{A. Radial consistency}

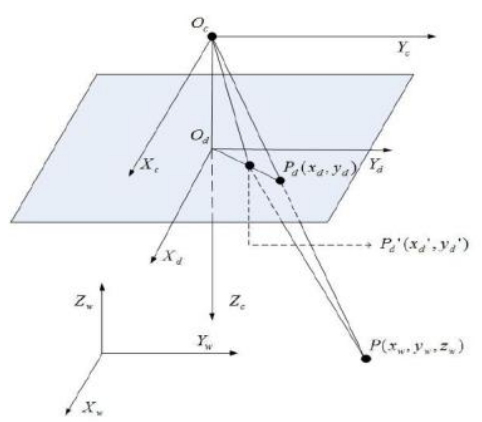

Figure 4.

As shown in Fig.4, the distortion of the camera lens does not change the center point and the image point of the camera. Thus, the following geometric relationships are always set up:

$$
O_{d} P_{d}^{\prime} / / P_{W} P
$$

According to the aforementioned coordinate system conversion relationship and formula (2), it can be obtained:

$$
\frac{x_{c}}{y_{c}}=\frac{x_{d}}{y_{d}}=\frac{r_{1} x_{w}+r_{2} y_{w}+r_{3} z_{w}+t_{x}}{r_{4} x_{w}+r_{5} y_{w}+r_{6} z_{w}+t_{y}}
$$

\section{B. Camera external parameter calibration}

Sorting out the formula (5): 


$$
x_{d}=\left[\begin{array}{lllllll}
x_{w} y_{d} & y_{w} y_{d} & z_{w} y_{d} & y_{d} & -x_{w} x_{d} & -y_{w} x_{d} & -z_{w} x_{d}
\end{array}\right]\left[\begin{array}{c}
\frac{r_{1}}{T_{y}} \\
\frac{r_{2}}{T_{y}} \\
\frac{r_{3}}{T_{y}} \\
\frac{r_{y}}{T_{y}} \\
\frac{r_{y}}{T_{y}} \\
T_{y} y_{d} \\
y_{w} y_{d} \\
r_{w} y_{d} \\
y_{d}
\end{array}\right.
$$

As it is known, that $x d$, yd can be converted by the image coordinate system. Then, when the number of feature points when $\mathrm{N}$ is greater than 7 , the only equation with the least squares solution, $\left[\begin{array}{lllllll}r_{1} / T_{y} & r_{2} / T_{y} & r_{3} / T_{y} & r_{4} / T_{y} & r_{5} / T_{y} & r_{6} / T_{y} & r_{7} / T_{y}\end{array}\right]^{T}$ can be obtained.

The value of Ty can be obtained by the constraint of the rotation matrix, and then the values of $\mathrm{r} 1, \mathrm{r} 2, \mathrm{Tx}, \mathrm{r} 4, \mathrm{r} 5$ are obtained. The orthogonality of the rotation matrix is obtained by r3,r6, r7, r8, r9.

This can be obtained by the rotation matrix $\mathrm{R}$ to solve the attitude of the aircraft, and through the translation matrix $\mathrm{T}$ to solve the aircraft position.

\section{Error analysis and landmark design}

Visual navigation error is mainly from two aspects, one is the image preprocessing of image filtering, edge detection and feature extraction of the image algorithm accuracy and high precision of the algorithm is bound to improve the accuracy.

In this paper, the Harris corner extraction algorithm is used in the corner extraction[9]. Firstly, the 2D gray image is $\mathrm{I}$, and the points $(\mathrm{u}, \mathrm{v})$ along the $\mathrm{x}$, the $\mathrm{y}$ is translated, and the sum of the squares is obtained.

$$
S(x, y)=\sum_{u} \sum_{v} w(u, v)(I(u+x, v+y)-I(u, v))^{2}
$$

Taylor expansion,

$$
I(u+x, v+y) \approx I(u, v)+I_{x}(u, v) \bullet x+I_{y}(u, v) \bullet y
$$

From formulas (7) and (8) available:

$$
S(x, y)=(x, y) \bullet A
$$

$$
\text { and } A=\sum_{u} \sum_{v} w(u, v)\left[\begin{array}{cc}
I_{x}^{2} & I_{x} I_{y} \\
I_{x} I_{y} & I_{y}^{2}
\end{array}\right]
$$

The Harris operator calculates the two eigenvalues of the matrix $A$, named $\lambda_{1}$ and $\lambda_{2}$, the point can make the $\lambda_{1} \approx 0$ and $\lambda_{2}$ for the larger positive point that is for the corner point.

There is also a class of errors caused by landmarks, unreasonable landmark will feature extraction of the failure, the lack of image information, cannot complete the solution. In the actual situation, the world coordinate system generally chooses the ground, so $\mathrm{Zw}$ in Eq. (6) are 0 . In theory, the relevant parameters can be solved only by 5 different points. Consider the design of cross landmark and $\mathrm{T}$ type landmark, different form of landmark, the corresponding feature points are different, respectively, simulation experiments, and compare their error results.

\section{KALMAN FILTER}

Kalman filter is a minimum variance estimation [10] using recursive algorithm, and it is designed by using state space method in time domain. In the navigation parameter estimation of UAV, the data processed by Kalman filter can be processed to improve the accuracy of data.

In order to improve the navigation precision of UAV landing, kalman filter is applied to process the data further. Select $\left[\begin{array}{lllllllll}X_{T} & Y_{T} & Z_{T} & \dot{X}_{T} & \dot{Y}_{T} & \dot{Z}_{T} & \ddot{X}_{T} & \ddot{Y}_{T} & \ddot{Z}_{T}\end{array}\right]^{T}$ as state variables, $\left[\begin{array}{lll}X_{T} & Y_{T} & Z_{T}\end{array}\right]^{T}$ as measurements. Then the system state equation can be got :

$\left[\begin{array}{c}X_{T}(k+1) \\ Y_{T}(k+1) \\ Z_{T}(k+1) \\ \dot{X}_{T}(k+1) \\ \dot{Y}_{T}(k+1) \\ \dot{Z}_{T}(k+1) \\ \ddot{X}_{T}(k+1) \\ \ddot{Y}_{T}(k+1) \\ \ddot{Z}_{T}(k+1)\end{array}\right]=\left[\begin{array}{ccccccccc}1 & 0 & 0 & T_{t} & 0 & 0 & T_{t}^{2} / 2 & 0 & 0 \\ 0 & 1 & 0 & 0 & T_{t} & 0 & 0 & T_{t}^{2} / 2 & 0 \\ 0 & 0 & 1 & 0 & 0 & T_{t} & 0 & 0 & T_{t}^{2} / 2 \\ 0 & 0 & 0 & 1 & 0 & 0 & T_{t} & 0 & 0 \\ 0 & 0 & 0 & 0 & 1 & 0 & 0 & T_{t} & 0 \\ 0 & 0 & 0 & 0 & 0 & 1 & 0 & 0 & T_{t} \\ 0 & 0 & 0 & 0 & 0 & 0 & 1 & 0 & 0 \\ 0 & 0 & 0 & 0 & 0 & 0 & 0 & 1 & 0 \\ 0 & 0 & 0 & 0 & 0 & 0 & 0 & 0 & 1\end{array}\right]\left[\begin{array}{c}X_{T}(k) \\ Y_{T}(k) \\ Z_{T}(k) \\ \dot{X}_{T}(k) \\ \dot{Y}_{T}(k) \\ \dot{Z}_{T}(k) \\ \ddot{X}_{T}(k) \\ \ddot{Y}_{T}(k) \\ \ddot{Z}_{T}(k)\end{array}\right]+\left[\begin{array}{c}0 \\ 0 \\ 0 \\ 0 \\ 0 \\ 0 \\ W_{X} \\ W_{Y} \\ W_{Z}\end{array}\right]$

(10)

Observation equation:

$$
\left[\begin{array}{c}
X_{T}(k+1) \\
Y_{T}(k+1) \\
Z_{T}(k+1)
\end{array}\right]=\left[\begin{array}{ccccccccc}
1 & 0 & 0 & 0 & 0 & 0 & 0 & 0 & 0 \\
0 & 1 & 0 & 0 & 0 & 0 & 0 & 0 & 0 \\
0 & 0 & 1 & 0 & 0 & 0 & 0 & 0 & 0
\end{array}\right]\left[\begin{array}{c}
X_{T}(k+1) \\
Y_{T}(k+1) \\
Z_{T}(k+1) \\
\dot{X}_{T}(k+1) \\
\dot{Y}_{T}(k+1) \\
\dot{Z}_{T}(k+1) \\
\ddot{X}_{T}(k+1) \\
\ddot{Y}_{T}(k+1) \\
\ddot{Z}_{T}(k+1)
\end{array}\right]+\left[\begin{array}{c}
V_{X} \\
V_{Y} \\
V_{Z}
\end{array}\right]
$$

$\mathrm{Tt}$ is the sampling time; $\mathrm{Wx}, \mathrm{Wy}, \mathrm{Wz}$, are the disturbances of jerk in $\mathrm{X}, \mathrm{Y}, \mathrm{Z}$ direction respectively. $\mathrm{Vx}$, $\mathrm{Vy}, \mathrm{Vz}$ are the position measurement errors in $\mathrm{X}, \mathrm{Y}, \mathrm{Z}$ direction respectively.

\section{SIMULATION EXPERIMENT}

\section{A. Camera parameter setting}

Using MATLAB to build the simulation camera to take pictures of the landmarks, the camera's internal parameters

matrix is set to $\mathrm{K}$, $[\mathrm{O}$ $K=\left[\begin{array}{ccc}260 & 0 & 400 \\ 0 & 260 & 400 \\ 0 & 0 & 1\end{array}\right]_{.260}$ 
pixel coordinate system. These two parameters are adjustable.

Fig. 5 and Fig. 6 is a picture of the landmarks from different angles and distances.

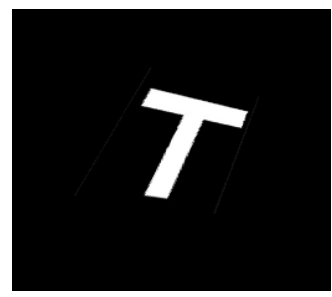

Figure 5.

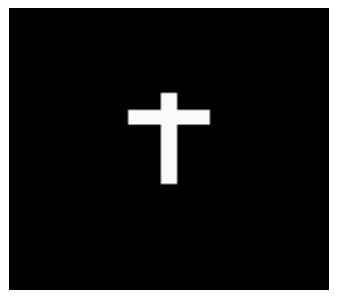

Figure 6.

\section{B. Harris corner extraction}

The corner points of the image are extracted by Harris, the result is Fig.7 and Fig.8.

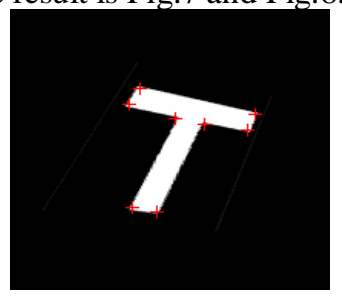

Figure 7.

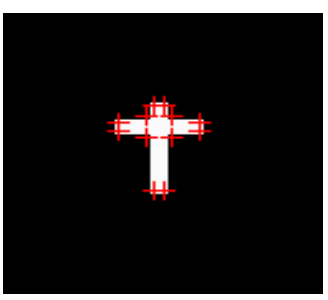

Figure 8.
There are three UAV positioning experiments, the first test using $\mathrm{T}$ landmark; the second test use of cross landmark; the third test will join the Kalman filter for processing data.

\section{Experimental results}

Experiments 1, the localization results of $\mathrm{T}$ type landmarks are shown in Fig.9, Fig.10 and Fig.11.

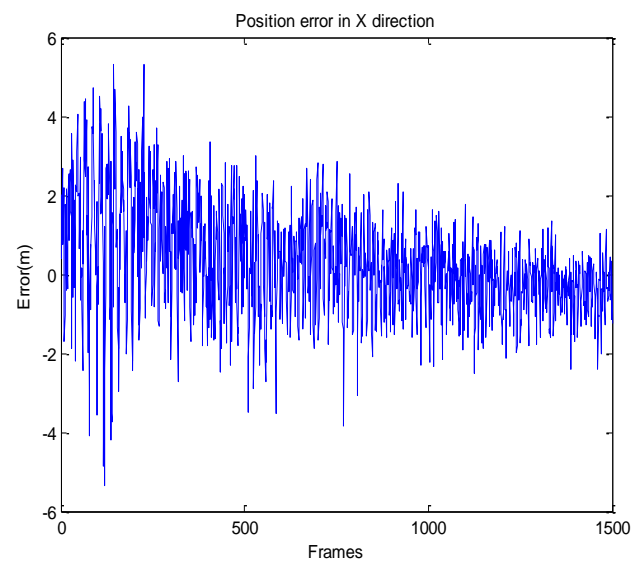

Figure 9. Position error of test 1 in $\mathrm{X}$ direction

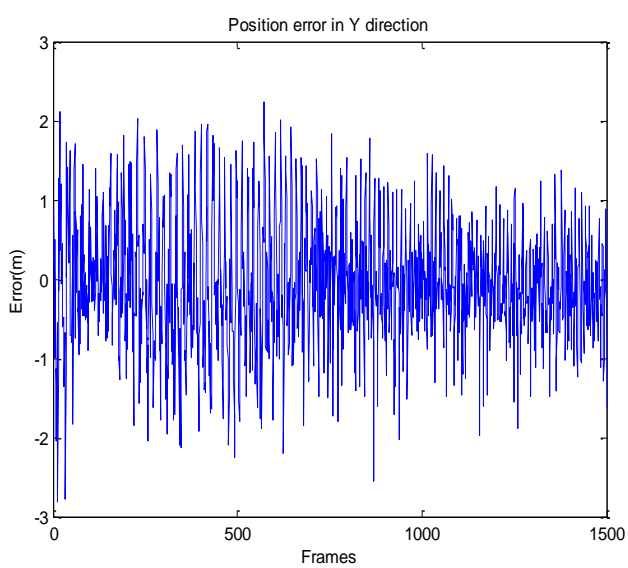

Figure 10. Position error of test 1 in Y direction

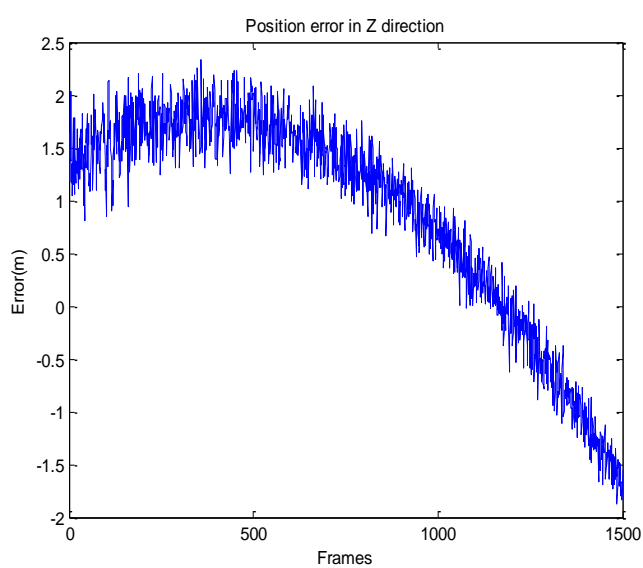

Figure 11. Position error of test 1 in $\mathrm{Z}$ direction

Experiment two, the localization results of the cross type landmarks are shown in Fig.12, Fig.13 and Fig. 14.

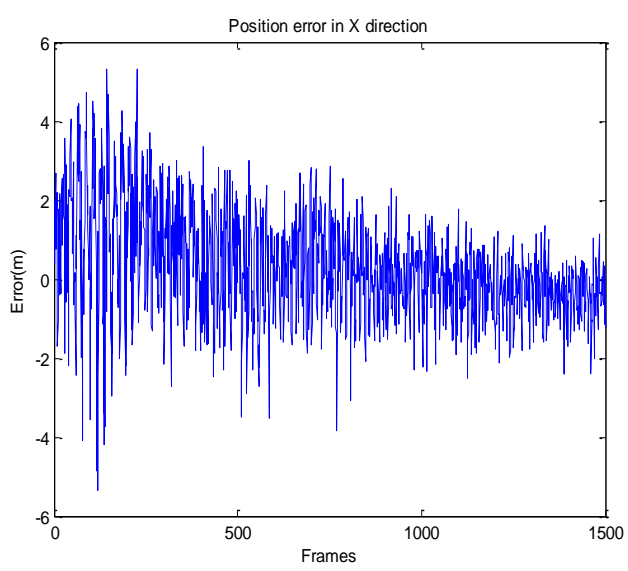

Figure 12. Position error of test 2 in $\mathrm{X}$ direction 


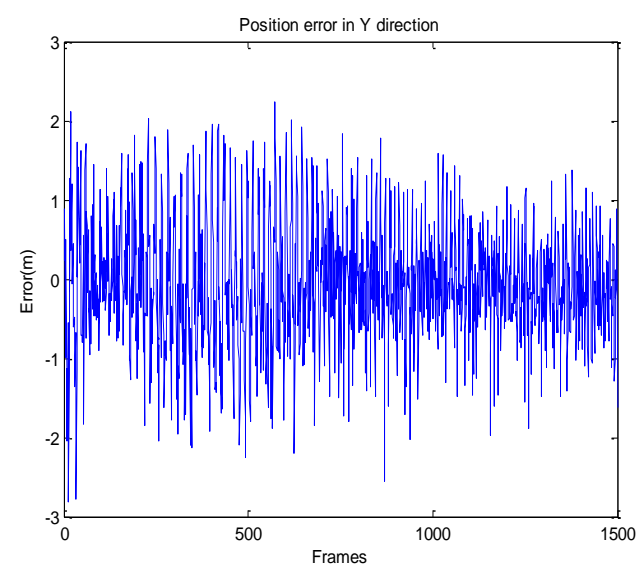

Figure 13. Position error of test 2 in $\mathrm{Y}$ direction

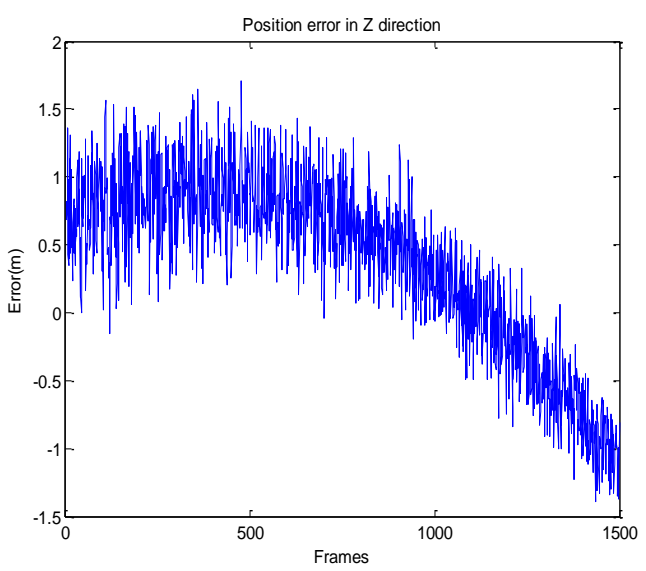

Figure 14. Position error of test 2 in $\mathrm{Z}$ direction

The position error of test 3 which has added Kalman filter for processing data in $X, Y, Z$ direction are presented in Fig.15, Fig.16 and Fig.17.

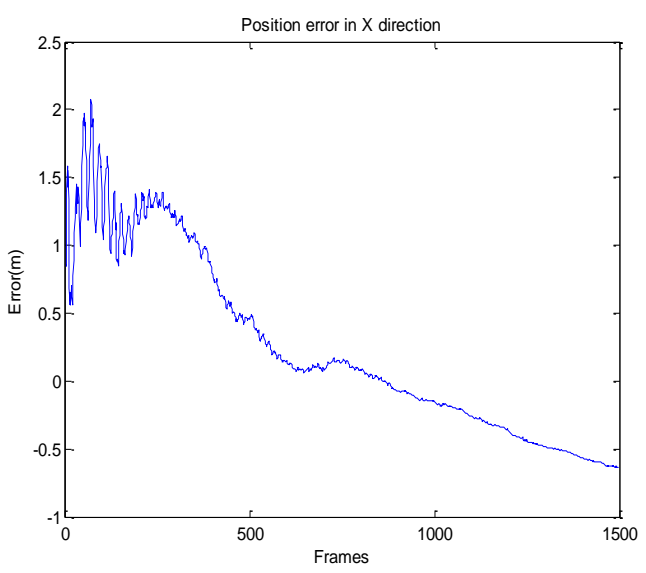

Figure 15. Position error of test 3 in $\mathrm{X}$ direction

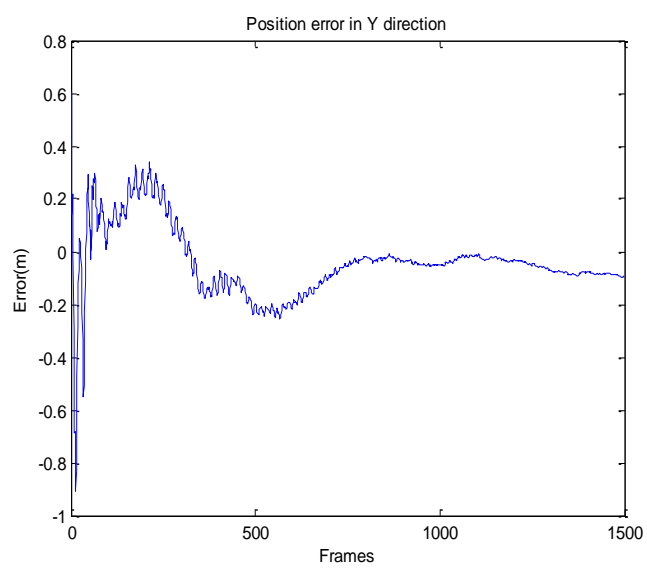

Figure 16. Position error of test 3 in Y direction

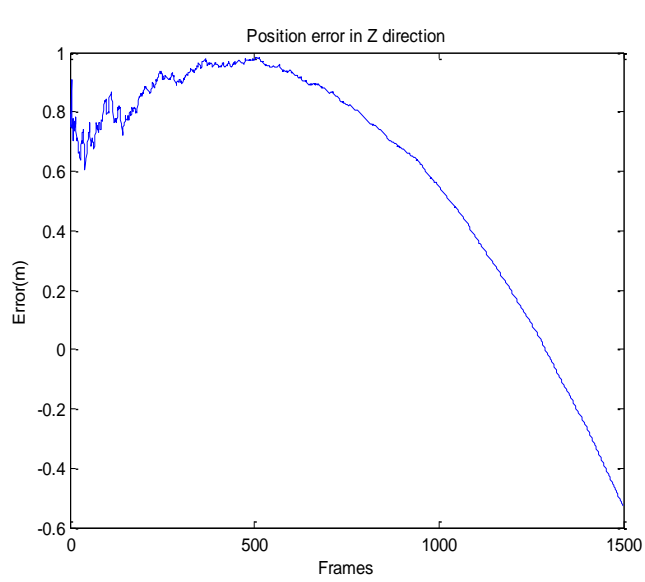

Figure 17. Position error of test 3 in $\mathrm{Z}$ direction

The root mean square errors of the three simulation tests are shown in Table 1.

TABLE I. ROOT MEAN SQUARE ERRORS IN X, Y, Z DIRECTIONS

\begin{tabular}{|c|c|c|c|}
\hline $\begin{array}{c}\text { Sequence } \\
\text { number }\end{array}$ & $\begin{array}{c}\mathrm{X} \\
\text { direction(meter) }\end{array}$ & $\begin{array}{c}\mathrm{Y} \\
\text { direction(meter) }\end{array}$ & $\begin{array}{c}\mathrm{Z} \\
\text { direction(meter) }\end{array}$ \\
\hline 1 & 1.4847 & 0.8785 & 1.3507 \\
\hline 2 & 1.4659 & 0.7885 & 0.7525 \\
\hline 3 & 0.7101 & 0.1601 & 0.7142 \\
\hline
\end{tabular}

By analyzing the results above, it can be concluded that:

The visual navigation model based on the principle of projection matrix has higher positioning accuracy. This is because in the near earth stage, the machine vision information is very high, and the projection matrix method has eliminated the interference of focal length variation.

Different types of landmark have different navigation and positioning accuracy. In a certain range, the positioning accuracy increases with the increase of the feature points.

After Kalman filtering, the positioning accuracy of the positioning of the direction $\mathrm{X}, \mathrm{Y}, \mathrm{Z}$ are certainly improved.

In conclusion, the positioning accuracy of visual navigation algorithm in this paper has high, which can meet the requirements of UAV landing. 


\section{CONCLUSION}

Firstly, the basic principle of visual navigation based on cooperative object is introduced.. Then, the pose parameter estimation algorithm is analyzed, and the different types of landmarks are proposed. By changing the feature points, the positioning accuracy is improved.. Finally, the data processing is processed by Calman filtering, and the navigation precision is improved.. Based on MATLAB software, a machine vision simulation model is built, and the visual navigation simulation experiment is conducted.. Experimental results show that the proposed method of visual navigation is very high and can meet the landing requirements of $\mathrm{UAV}$.

\section{REFERENCES}

[1] JIANG Xueming. The Present and Future of Sophisticated Approach and Landing System [J]. Avionics Technology. 1996.3, 18-21

[2] LI Sihai, HUI Pengzhou, FENG Peide. INS/DGPS Guide System for Aircraft Precision Approach and Landing [J]. Journal of Chinese Inertial Technology. 2000.8(1), 16-20.
[3] YANG Liang .The Landing Guide Technology for UAV [J]. Radio Engineering of China. 2009, 39(12), 29-30.

[4] MA Songde, Zhang Zhengyou. Computer visual [M]. Beijing: Science Press, 1998.

[5] Doehler H U. Korn B. Robust Position Estimation Using Images from an Uncalibrated Camera[C]. Proceedings of the 22nd Digital Avionics Systems Conference, 2003, 2: 1-7.

[6] DIAO Can, WANG Yingxun,JIN Ti.Machine vision aided technology for automatic landing of unmanned aerial vehicles. Chinese Journal of aviation (ET 2008: S1): 79-84.

[7] Yang Gui, Pengyu Guo, Hongliang Zhang, Airborne VisionBased Navigation Method for UAV Accuracy Landing Using Infrared Lamps[J].Intell Robot Syst 2013 (72) : 197-218.

[8] LIU Xunliang. Optical visual sensing [M]. Beijing: China Science and Technology Press, 1998 .

[9] WANG Wai, TANG Yiping, REN Juanli. An improved Harris corner extraction algorithm for [J]. optical precision engineering, 2008,16 (10): 1995-2001.

[10] QIN Yongyuan, WANG Shuhua. Kalman filter and integrated navigation principle, Northwestern Polytechnical University press, 2010,33 . 